\title{
Coupling of estuarine benthic and pelagic food webs to land-derived nitrogen sources in Waquoit Bay, Massachusetts, USA
}

\author{
Paulina Martinetto $^{1,2, *}$, Mirta Teichberg ${ }^{2}$, Ivan Valiela ${ }^{2}$ \\ ${ }^{1}$ Departamento de Biología, Universidad Nacional de Mar del Plata, CC573 Correo Central, \\ B7600WAG Mar del Plata, Argentina \\ ${ }^{2}$ Boston University Marine Program, Marine Biological Laboratory, 7 MBL Street, Woods Hole, Massachusetts 02543, USA
}

\begin{abstract}
The fact that land-derived sources of nutrients promote eutrophication in the receiving coastal waters implies coupling between land and marine environments. Increasing nitrogen inputs in the estuaries are followed by major shifts in biota composition and abundances. In the present paper we used $\mathrm{N}$ and $\mathrm{C}$ isotopic ratios to analyze the coupling of benthic and pelagic components of food webs to estuaries receiving different $\mathrm{N}$ loads from their watersheds. We found that primary producers, benthic taxa, and fishes were coupled to the watersheds and estuaries where they were collected. In contrast, zooplankton was uncoupled. Primary consumers and predators feeding on benthic prey within the estuaries were also coupled to the watershed and estuaries, but predators feeding on zooplankton were not. We hypothesized that short water residence time in these estuaries uncoupled plankton from terrestrial influence. Stable isotopic measurements of $\mathrm{N}$ in producers, consumers, POM, and sediment in different estuaries of Waquoit Bay, Massachusetts, USA, demonstrate a consistent link between land-use on contributing watersheds and the isotopic ratio in all the benthic components and food webs. The remarkably consistent link suggests that the benthos was tightly coupled to land-derived inputs, and that these components, particularly macrophytes, could be good indicators for monitoring increases in land-derived $\mathrm{N}$ inputs. Our results showed that stable isotopes of $\mathrm{N}$ and $\mathrm{C}$ have the potential for use in basic research and applied monitoring, but need to be applied considering the features of estuaries that might couple or uncouple organisms regarding dependency on land, such as hydrodynamic exchanges.
\end{abstract}

KEY WORDS: Eutrophication · Benthic coupling $\cdot$ Pelagic coupling $\cdot$ Land-estuary coupling $\cdot$ Food webs · Estuaries · Waquoit Bay

\section{INTRODUCTION}

Eutrophication is a major agent of change to coastal habitats worldwide (GESAMP 1990, National Research Council 1994). Increasing population densities along coastlines and the use of fertilizers have produced higher nutrient loading, which in turn has increased primary production, leading to major shifts in the species composition and abundances of flora and fauna living in estuaries (Valiela et al. 1997, Grall \& Chau- vaud 2002), as well as eutrophication in estuaries and coastal waters (Nixon 1995, Cloern 2001). The fact that land-derived sources of nutrients create eutrophication in receiving coastal waters implies considerable coupling due to the flow of material between land and estuarine environments.

Stable isotopes have been used to detect landderived sources of $\mathrm{N}$ (McClelland et al. 1997, McClelland \& Valiela 1998b, Wigand et al. 2001). Wastewater, fertilizers, and atmospheric $\mathrm{N}$, for example, 
have different $\delta^{15} \mathrm{~N}$ values, and hence the $\mathrm{N}$ exported from a given watershed bears stable isotopic signatures characterized by the land use on the watershed.

Stable isotopes have also been used to define food webs within estuaries (Peterson \& Fry 1987). Because elements such as carbon and nitrogen fractionate in predictable ways in aquatic organisms, it is possible to construct food webs based on these data. The ratio of ${ }^{15} \mathrm{~N}$ to ${ }^{14} \mathrm{~N}$ is used to determine trophic position. Consumers become enriched in $\delta^{15} \mathrm{~N}$ relative to their food by 3 to $4 \%$ (Michener \& Schell 1994). As a result of this stepwise trophic level enrichment, nitrogen stable isotopes have become a valuable tool in food web analysis.

The estuaries of Waquoit Bay, Massachusetts, USA, offer the opportunity to examine the isotopic response of estuarine flora and fauna to increased $\mathrm{N}$ loads at the watershed-estuarine scale. Estuaries that enter Waquoit Bay are similar in depth and water residence time, but they differ in the degree of urbanization and land use and hence in the $\mathrm{N}$ load received (Valiela et al. 2000, 2004). Studies carried out in these estuaries have shown a strong correlation between the $\delta^{15} \mathrm{~N}$ of estuarine biota and the relative contribution by wastewater to the land-derived $\mathrm{N}$ load entering the estuaries (McClelland et al. 1997, McClelland \& Valiela 1998a).

Thus, land use practices on watersheds may alter $\mathrm{N}$ loads, and therefore the components of the food web may incorporate the $\delta^{15} \mathrm{~N}$ signal of local watershedderived $\mathrm{N}$ sources. Species that live in the benthos have to sustain conditions across time; pelagic species, in contrast, either move advectively with estuarine circulation, or swim. These quite different exposures to ambient conditions may determine how likely it is that organisms in the benthos or plankton are reliable indicators of land-derived nutrient inputs. In turn, the linkage of isotopic values of organisms and watershedderived inputs might reveal a coupling between land use and estuarine food webs. In this paper, we use C and $\mathrm{N}$ isotope signatures of primary producers and consumers of estuaries in the Waquoit Bay system to ascertain which components of benthic and pelagic food webs are coupled to estuaries receiving different $\mathrm{N}$ loads from their watersheds.

\section{MATERIALS AND METHODS}

Study site. To define the coupling between land and the benthic and pelagic food web components in estuaries we measured $\mathrm{N}$ and $\mathrm{C}$ stable isotopes in benthic invertebrates, juvenile fishes, major taxa of zooplankton, macrophytes, particulate organic matter (POM), and sediment from 3 estuaries that receive different nitrogen loads. These estuaries have similar water residence times (approximately 1 to $2 \mathrm{~d}$ ), and range in salinity from 0 to $32 \mathrm{ppt}$. They receive relatively low (Sage Lot Pond), intermediate (Quashnet River), and high (Childs River) nitrogen loads of 14, 350, and $600 \mathrm{~kg} \mathrm{~N} \mathrm{ha}^{-1} \mathrm{yr}^{-1}$, respectively (Valiela et al. 1997). N loads to Waquoit Bay include atmospheric deposition, fertilizer use, and wastewater disposal. Wastewater contributes $~ 50 \%$ of the total $\mathrm{N}$ load and $75 \%$ of the anthropogenic (wastewater + fertilizer) $\mathrm{N}$ load to the bay (Valiela et al. 1997). The $\delta^{15} \mathrm{~N}$ values of groundwater dissolved inorganic nitrogen (DIN) entering estuaries increase with the increased $\mathrm{N}$ load of the system (Childs River 9.5, Quashnet River 5.8, Sage Lot Pond 0.5, McClelland \& Valiela 1998b). Producers within the estuaries appear to integrate the stable $\mathrm{N}$ signatures from groundwater reflecting the proportion of $\mathrm{N}$ delivered to the estuary from wastewater inputs (McClelland \& Valiela 1998b). These differences make it possible to assess the response of estuarine organisms to different degrees of loading, with different isotopic signatures. Therefore $\delta^{15} \mathrm{~N}$ signatures can be used to indicate coupling of food web components to the watersheds and estuaries. We sampled organisms, POM, and sediment at 3 stations in Sage Lot Pond (SLP) and at 5 stations in Quashnet River (QR) and Childs River (CR) (Fig. 1). Sampling stations were arranged in transects along the estuaries to capture the variation across the estuaries.

Coupling of primary producers and benthic and pelagic organisms to land-derived $\mathbf{N}$ sources. To assess coupling between land and the producers of the benthic and pelagic food webs within the Waquoit Bay estuaries, we sampled POM, sediment, and macrophytes from each site. We refer to POM as a 'producer' because it is composed largely of organic matter from producers and because the $\delta^{15} \mathrm{~N}$ of POM in Waquoit Bay is primarily influenced by phytoplankton (Yelenik et al. 1996, McClelland \& Valiela 1998b). We collected water near the water-sediment interface and at $0.5 \mathrm{~m}$ below the surface at every site and filtered it through Whatman GF/C glass fiber filters (effective pore size $\approx 1.2 \mu \mathrm{m}$ ) to obtain the POM. Surface sediment was collected from the benthic dredge samples (see benthic collection method described below) and was treated with $1.0 \mathrm{~N} \mathrm{HCl}$ to remove bicarbonate. Macrophytes were collected by hand at each site and cleaned with deionized water.

To determine the coupling between land and benthic and pelagic fauna, we sampled organisms from both the benthos and the water column. We collected benthic invertebrates by taking 3 Ekman dredges at each site from the 3 subestuaries. Contents of the dredges were rinsed through a $0.5 \mathrm{~mm}$ sieve; invertebrates were sorted by species. We used a $5 \mathrm{~m}$ seine $(1 \mathrm{~cm}$ mesh size $)$ to collect fish, shrimps, and crabs 


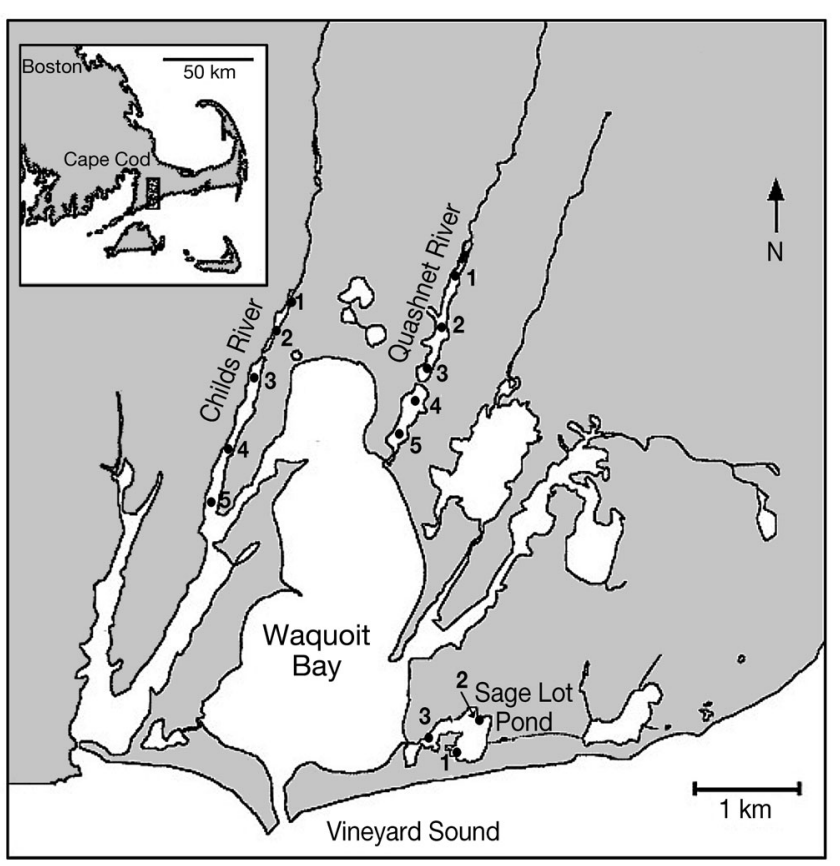

Fig. 1. Waquoit Bay map showing sampling sites at Childs River, Quashnet River, and Sage Lot Pond

along the shoreline. Ascidians were collected by hand. All organisms were washed with deionized water. In the case of fishes and holothuroids, the digestive tract was removed, and the remaining muscle washed with deionized water. The crabs were maintained $24 \mathrm{~h}$ in filtered seawater for gastric evacuation. Zooplankton were collected at each site by towing a zooplankton net $(12 \mathrm{~cm}$ diameter, $45 \mathrm{~cm}$ length, mesh size $=60 \mu \mathrm{m}$ ) against the current for $20 \mathrm{~min}$. The major taxa were sorted by developmental stage, rinsed with deionized water, and collected on Whatman filters. Acartia tonsa was the dominant copepod found in all 3 estuaries. We measured $\delta^{15} \mathrm{~N}$ and $\delta^{13} \mathrm{C}$ of adult, copepodid, and nauplius stages of this species. We also measured the $\delta^{15} \mathrm{~N}$ and $\delta^{13} \mathrm{C}$ of polychaete larvae found in the estuaries. The zooplankton samples for isotope analysis were composites of 20 to 200 individuals. Specimens of the ctenophore Mnemiopsis leidyi were collected by hand and rinsed with deionized water before analysis.

Producer and consumer samples were dried at $55^{\circ} \mathrm{C}$, ground to fine powder, weighed, and loaded into tin capsules. Seston and zooplankton samples were kept in pre-weighed filters, weighed, and then loaded into tin capsules. Isotope analyses were performed by mass spectrometer in the 'Stable Isotope Facility' of the University of California at Davis (USA). Results are reported as comparisons with atmospheric nitrogen (for N) and Vienna Pee Dee Belemnite (for C) as standards and calculated as: $\delta^{13} \mathrm{C}$ or $\delta^{15} \mathrm{~N}(\%)=\left[\left(R_{\text {sample }}-R_{\text {standard }}\right) / R_{\text {standard }}\right] \times 10^{3}$

where $R$ is $\left({ }^{15} \mathrm{~N} /{ }^{14} \mathrm{~N}\right)$ or $\left({ }^{13} \mathrm{C} /{ }^{12} \mathrm{C}\right)$. Duplicate determinations on the same sample usually differed by $<0.2 \%$.

To determine if the isotopic signature of various taxonomic groups of benthic and pelagic organisms was correlated to the $\mathrm{N}$ load and percentage of wastewater contribution from the subwatersheds emptying into the estuary in which the taxa were collected, we pooled species into broad taxonomic groups. The relationship of $\delta^{15} \mathrm{~N}$ signatures of primary producers and organisms vs. $\mathrm{N}$ load was described by linear regression analysis (Type I regression, Neter et al. 1985). The $\delta^{15} \mathrm{~N}$ signatures of primary producers were also regressed to wastewater percentage in each estuary. To examine whether the different taxonomic groups differed in their relationships to nitrogen load, we compared slopes using Student's $t$-tests (Devore 2000). To have a more comprehensive data set in this paper, we included isotopic data collected for this study as well as data from previous studies done in these estuaries (Hauxwell et al. 1998, McClelland \& Valiela 1998a, Griffin \& Valiela 2001, Shriver et al. 2002, Carmichael 2003).

Nutrient sources and trophic level effects on $\delta^{15} \mathbf{N}$ signature. The isotopic signature of any species may depend, not only on the $\mathrm{N}$ source, but also on the trophic position of the species. To distinguish the relative importance of nitrogen source and trophic level on the $\delta^{15} \mathrm{~N}$ isotope values of the organisms, we grouped the species as grazers, filter feeders, deposit feeders, scavengers, and predators. The assignation of feeding habit was based on a review of literature (Appendix 1). The $\delta^{15} \mathrm{~N}$ signatures of each taxon assigned to each feeding type were then averaged for each estuary. To assess the coupling of the mean isotopic signature and the nitrogen load entering estuaries, we regressed (Type I regression, Neter et al. 1985) these variables. To examine whether the different trophic groups differed in their relationships to the nitrogen load, we compared slopes using $t$-tests (Devore 2000); where differences between slopes were not found, the intercepts were compared using analysis of covariance (ANCOVA) (Sokal \& Rohlf 2003) to determine the enrichment in ${ }^{15} \mathrm{~N}$ from food types to consumers.

\section{RESULTS AND DISCUSSION}

In this section we first analyze the relationships of the $\mathrm{N}$ load and wastewater percentage to the isotopic signatures of taxa and trophic groups. Then we examine the relationships between producers and consumers in each estuary and define differences in $\delta^{15} \mathrm{~N}$ 
Table 1. $\delta^{13} \mathrm{C}$ and $\delta^{15} \mathrm{~N}$ signatures (mean $\pm \mathrm{SE}$ ) for macrophytes, sediment, particulate organic matter (POM), and benthic and pelagic consumers for Sage Lot Pond, Quashnet River, and Childs River. Values are from this study and/or other sources (1: present study; 2: McClelland \& Valiela 1998; 3: Shriver et al. 2002; 4: Carmichael 2003; 5: Griffin \& Valiela 2001). Blank spaces: no data

\begin{tabular}{|c|c|c|c|c|c|c|c|c|c|c|c|c|c|}
\hline \multirow[t]{2}{*}{ Species } & \multicolumn{4}{|c|}{ Sage Lot Pond } & \multicolumn{4}{|c|}{ Quashnet River —_ } & \multirow[b]{2}{*}{$\delta^{13} \mathrm{C}$} & \multicolumn{3}{|c|}{ Childs River } & \multirow[t]{2}{*}{ Source } \\
\hline & $\delta^{13} \mathrm{C}$ & $\mathrm{n}$ & $\delta^{15} \mathrm{~N}$ & $\mathrm{n}$ & $\delta^{13} \mathrm{C}$ & $\mathrm{n}$ & $\delta^{15} \mathrm{~N}$ & $\mathrm{n}$ & & $\mathrm{n}$ & $\delta^{15} \mathrm{~N}$ & $\mathrm{n}$ & \\
\hline \multicolumn{14}{|l|}{ Macrophytes } \\
\hline Cladophora vagabunda & -15.30 & 1 & 3.40 & 1 & $-14.76 \pm 0.62$ & 2 & $4.34 \pm 0.49$ & 2 & $-14.73 \pm 0.81$ & 2 & $6.52 \pm 1.59$ & 2 & 1,2 \\
\hline Codium fragile & -12.28 & 1 & 5.85 & 1 & -13.03 & 1 & 6.10 & 1 & -13.23 & 1 & 8.19 & 1 & 1 \\
\hline Enteromorpha sp. & $-19.10 \pm 0.21$ & 2 & $4.90 \pm 0.10$ & 2 & $-17.70 \pm 0.60$ & 2 & $6.40 \pm 0.30$ & 2 & $-18.70 \pm 0.20$ & 2 & $8.40 \pm 0.20$ & 2 & 2 \\
\hline Fucus vesiculosus & -14.60 & 1 & 4.59 & 1 & -14.48 & 1 & 5.86 & 1 & -12.91 & 1 & 7.90 & 1 & 1 \\
\hline Gracilaria tikvahiae & -19.50 & 1 & 5.10 & 1 & $-16.87 \pm 0.15$ & 3 & $6.16 \pm 0.17$ & 3 & $-15.94 \pm 0.62$ & 3 & $8.03 \pm 0.28$ & 3 & 1,2 \\
\hline Ulva lactuca & $-11.25 \pm 0.14$ & 4 & $3.82 \pm 0.31$ & 4 & -6.54 & 1 & 7.91 & 1 & $-6.46 \pm 1.15$ & 2 & $9.28 \pm 0.7$ & 2 & 1 \\
\hline Spartina alterniflora & $-13.26 \pm 0.06$ & 2 & $3.28 \pm 0.49$ & 2 & $-12.70 \pm 0.22$ & 3 & $7.79 \pm 0.81$ & 3 & $-13.35 \pm 0.06$ & 2 & $8.15 \pm 0.21$ & 2 & 1 \\
\hline Zostera marina & -10.26 & 1 & 1.99 & 1 & & & & & & & & & \\
\hline Sediment & $-14.94 \pm 0.33$ & 6 & $2.19 \pm 0.36$ & 6 & $-21.45 \pm 1.75$ & 6 & $4.27 \pm 0.33$ & 6 & $-19.36 \pm 0.74$ & 9 & $4.94 \pm 0.12$ & 9 & 1 \\
\hline \multicolumn{14}{|l|}{ POM } \\
\hline Surface & $-20.24 \pm 1.24$ & 6 & $3.70 \pm 1.32$ & 6 & $-20.69 \pm 0.70$ & 5 & $5.20 \pm 0.52$ & 5 & $-20.06 \pm 1.00$ & 5 & $6.27 \pm 1.10$ & 5 & 1 \\
\hline Bottom & $-17.48 \pm 0.64$ & 2 & $5.17 \pm 1.08$ & 2 & $-19.33 \pm 1.35$ & 3 & $5.29 \pm 1.20$ & 3 & $-19.91 \pm 0.28$ & 3 & $5.29 \pm 1.36$ & 3 & 1 \\
\hline \multicolumn{14}{|l|}{ Amphipoda } \\
\hline Cymadusa compta & $-14.40 \pm 0.30$ & 2 & $4.10 \pm 0.20$ & 2 & & & & & $-16.00 \pm 0.40$ & 2 & $7.60 \pm 0.20$ & 2 & 2 \\
\hline Gammarus mucronatus & $-13.63 \pm 0.81$ & 2 & $6.69 \pm 0.97$ & 2 & & & & & $-14.80 \pm 0.40$ & 2 & $7.80 \pm 0.30$ & 2 & 2 \\
\hline Gammarus oceanicus & & & & & -15.49 & 1 & 5.39 & 1 & & & & & 1 \\
\hline Microdeutopus gryllotalpa & $-13.40 \pm 0.10$ & 2 & $3.80 \pm 0.10$ & 2 & & & & & $-14.80 \pm 0.40$ & 2 & $6.60 \pm 0.10$ & 2 & 2 \\
\hline \multicolumn{14}{|l|}{ Ascidiacea } \\
\hline Molgula manhattensis & & & & & -16.70 & 1 & 7.24 & 1 & $-18.19 \pm 1.04$ & 2 & $7.16 \pm 1.34$ & 2 & 1 \\
\hline \multicolumn{14}{|l|}{ Bivalvia } \\
\hline Argopecten irradians & & & $6.68 \pm 0.38$ & 2 & & & 6.81 & 1 & & & $8.18 \pm 0.11$ & 2 & 3 \\
\hline Geukensia demissa & -19.00 & 1 & $5.76 \pm 0.60$ & 4 & & & $7.50 \pm 0.19$ & 3 & -18.50 & 1 & $8.18 \pm 0.38$ & 4 & 2 \\
\hline Mercenaria mercenaria & $-16.40 \pm 0.30$ & & $6.70 \pm 0.02$ & & -17.90 & 1 & 8.20 & 1 & $-15.90 \pm 0.20$ & & $9.50 \pm 0.06$ & & 4 \\
\hline Mya arenaria & $-16.50 \pm 0.10$ & & $6.80 \pm 0.04$ & & -17.50 & 1 & 8.01 & 1 & $-16.90 \pm 0.20$ & & $10.10 \pm 0.07$ & & 4 \\
\hline \multicolumn{14}{|l|}{ Decapoda } \\
\hline Callinectes sapidus & & & & & $-11.60 \pm 0.64$ & 3 & $7.99 \pm 0.72$ & 3 & $-14.18 \pm 0.56$ & 3 & $9.98 \pm 0.07$ & 3 & 1 \\
\hline Carcinus maenas & $-12.72 \pm 0.86$ & 2 & $6.92 \pm 0.63$ & 2 & $-12.76 \pm 0.09$ & 2 & $10.00 \pm 0.38$ & 2 & & & & & 1 \\
\hline Crangon septemspinosa & -13.36 & 1 & 6.82 & 1 & $-12.64 \pm 0.15$ & 2 & 9.01 & 2 & $-14.08 \pm 0.84$ & 3 & $9.27 \pm 1.21$ & 3 & 1 \\
\hline Hypolite zostericola & & & $6.69 \pm 0.31$ & 2 & & & & & & & & & 1 \\
\hline Libinia dubia & -13.29 & 1 & 7.60 & 1 & & & & & & & & & 1 \\
\hline Neopanopeus sayi & $-12.18 \pm 0.30$ & 2 & $6.04 \pm 0.59$ & 2 & -1058 & 1 & 6.65 & 1 & & & & & 1 \\
\hline Pagurus longicarpus & & & & & & & & & -10.67 & 1 & 8.18 & 1 & 1 \\
\hline Palaemonetes pugio & $-13.56 \pm 0.36$ & 2 & $7.17 \pm 0.49$ & 2 & $-13.27 \pm 0.18$ & 3 & $9.28 \pm 0.08$ & 3 & $-14.75 \pm 0.23$ & 4 & $10.50 \pm 0.24$ & 4 & 1 \\
\hline Palaemonetes vulgaris & -11.24 & 1 & 8.82 & 1 & -12.83 & 1 & 9.73 & 1 & -14.90 & 1 & 10.74 & 1 & 1,2 \\
\hline Rhitripanopeus harssi & -12.36 & 1 & 6.02 & 1 & $-11.83 \pm 0.29$ & 2 & $7.69 \pm 1.33$ & 2 & & & & & 1 \\
\hline Gastropoda & & & & & & & & & & & & & \\
\hline Crepidula fornicata & -17.63 & 1 & 7.42 & 1 & & & & & & & & & 1 \\
\hline Haminoea solitaria & & & & & -12.07 & 1 & 6.16 & 1 & $-12.74 \pm 0.28$ & 2 & $7.82 \pm 0.67$ & 2 & 1 \\
\hline Nassarius obsoletus & -12.64 & 1 & 6.09 & 1 & -11.42 & 1 & 6.46 & 1 & $-13.65 \pm 0.55$ & 3 & $9.54 \pm 0.19$ & 3 & 1 \\
\hline Holothuroidea & & & & & & & & & & & & & \\
\hline Leptosynapta tenuis & $-12.99 \pm 0.49$ & 2 & $7.89 \pm 1.81$ & 2 & $-13.77 \pm 0.52$ & 2 & $8.80 \pm 0.64$ & 2 & & & & & 1 \\
\hline Sclerodactyla briareus & & & 7.41 & 1 & $-13.18 \pm 0.18$ & 2 & $9.78 \pm 1.20$ & 2 & $-14.70 \pm 1.18$ & 3 & $11.47 \pm 1.01$ & 3 & 1 \\
\hline Isopoda & & & & & & & & & & & & & \\
\hline Erichsonella filiformis & $-13.60 \pm 1.80$ & 2 & $4.30 \pm 1.00$ & 2 & & & & & $-14.50 \pm 0.60$ & 2 & $7.70 \pm 0.80$ & 2 & 2 \\
\hline Cyathura polita & -12.79 & 1 & 6.08 & 1 & $-12.96 \pm 0.93$ & 2 & $10.96 \pm 0.43$ & 2 & $-12.74 \pm 0.85$ & 2 & $11.61 \pm 0.38$ & 2 & 1 \\
\hline Polychaeta & & & & & & & & & & & & & \\
\hline Aglaophamus circinata & & & & & -22.37 & 1 & 10.74 & 1 & & & & & 1 \\
\hline Arabella iridicolor & $-14.71 \pm 0.13$ & 2 & $8.72 \pm 1.63$ & 2 & & & & & & & & & 1 \\
\hline Cyrratulus grandis & -14.82 & 1 & 4.67 & 1 & & & & & & & & & 1 \\
\hline Clymenella torquata & & & & & & & & & -15.85 & 1 & 6.89 & 1 & 1 \\
\hline Etone lactea & & & & & -12.63 & 1 & 9.17 & 1 & & & & & 1 \\
\hline Glycera americana & -13.21 & 1 & 7.46 & 1 & & & & & $-16.49 \pm 1.85$ & 2 & $12.15 \pm 0.34$ & 1 & 1 \\
\hline Harmathoe extenuata & -14.98 & 1 & 8.72 & 1 & & & & & & & & & 1 \\
\hline Harmathoe imbricata & -14.52 & 1 & 7.97 & 1 & & & & & & & & & 1 \\
\hline Heteromastus filiformis & -13.40 & 1 & 5.81 & 1 & & & & & & & & & 1 \\
\hline Lumbrinereis fragilis & & & & & & & & & $-14.96 \pm 0.61$ & 2 & $10.22 \pm 1.45$ & 2 & 1 \\
\hline Neanthes succinea & & & & & -13.48 & 1 & 8.47 & 1 & -14.75 & 1 & 8.00 & 1 & 1 \\
\hline Notomastus latericeus & & & & & -21.53 & 1 & 8.89 & 1 & $-18.43 \pm 1.87$ & 2 & $10.32 \pm 0.33$ & 2 & 1 \\
\hline
\end{tabular}


Table 1 (continued)

\begin{tabular}{|c|c|c|c|c|c|c|c|c|c|c|c|c|c|}
\hline \multirow[t]{2}{*}{ Species } & \multirow[b]{2}{*}{$\delta^{13} \mathrm{C}$} & \multicolumn{3}{|c|}{ Sage Lot Pond — } & \multicolumn{4}{|c|}{ Quashnet River _- } & \multirow[b]{2}{*}{$\delta^{13} \mathrm{C}$} & \multicolumn{3}{|c|}{ Childs River } & \multirow[t]{2}{*}{ Source } \\
\hline & & $\mathrm{n}$ & $\delta^{15} \mathrm{~N}$ & $\mathrm{n}$ & $\delta^{13} \mathrm{C}$ & $\mathrm{n}$ & $\delta^{15} \mathrm{~N}$ & $\mathrm{n}$ & & $\mathrm{n}$ & $\delta^{15} \mathrm{~N}$ & $\mathrm{n}$ & \\
\hline \multicolumn{14}{|l|}{ Polychaeta (continued) } \\
\hline Orbinia ornata & $-14.34 \pm 1.07$ & 2 & $4.84 \pm 0.33$ & 2 & $-13.98 \pm 0.16$ & 2 & $7.31 \pm 0.45$ & 2 & & & & & 1 \\
\hline Pectinaria gouldii & & & & & & & & & -16.24 & 1 & 6.75 & 1 & 1 \\
\hline Podarke obscura & -13.74 & 1 & 8.15 & 1 & $-12.73 \pm 0.65$ & 2 & $9.20 \pm 0.15$ & 2 & -15.36 & 1 & 10.33 & 1 & 1 \\
\hline Polycirrus eximius & -16.57 & 1 & 4.38 & 1 & & & & & -15.72 & 1 & 8.17 & 1 & 1 \\
\hline Sabellaria vulgaris & & & & & & & & & -14.94 & 1 & 9.24 & 1 & 1 \\
\hline Tharyx acutus & -14.08 & 2 & 4.97 & 2 & & & & & & & & & 1 \\
\hline \multicolumn{14}{|l|}{ Teleostei } \\
\hline Anguilla rostrata & & & & & $-14.40 \pm 0.08$ & 2 & $10.27 \pm 0.30$ & 2 & & & & & 1 \\
\hline Cyprinodon variegatus & $-11.50 \pm 0.90$ & 3 & $5.20 \pm 0.50$ & 3 & & & & & $-14.00 \pm 0.50$ & 3 & $9.80 \pm 0.30$ & 3 & 2 \\
\hline Fundulus heteroclitus & $-14.00 \pm 0.40$ & 3 & $8.70 \pm 0.32$ & 3 & -14.64 & 1 & $10.95 \pm 0.24$ & 15 & $-14.60 \pm 0.50$ & 3 & $11.64 \pm 0.27$ & 3 & 2,5 \\
\hline Fundulus majalis & & & & & $-14.49 \pm 0.48$ & 2 & $10.30 \pm 0.40$ & 2 & & & & & 1 \\
\hline Gasterosteus aculeatus & -16.90 & 1 & 9.00 & 1 & -14.52 & 1 & 11.39 & 1 & $-15.40 \pm 0.40$ & 3 & $12.30 \pm 0.40$ & 3 & 1,2 \\
\hline Gobiosoma bosc & & & & & $-13.77 \pm 0.20$ & 2 & $11.25 \pm 0.20$ & 2 & & & & & 1 \\
\hline Menidia menidia & $-16.50 \pm 0.50$ & 4 & $9.02 \pm 0.11$ & 11 & -13.81 & 1 & $10.64 \pm 0.15$ & 16 & $-17.70 \pm 0.80$ & 4 & $11.33 \pm 0.18$ & 14 & 2,5 \\
\hline $\begin{array}{l}\text { Pseudopleuronectes } \\
\text { americanus }\end{array}$ & & & & & -14.37 & 1 & 9.21 & 1 & & & & & 1 \\
\hline Tautogolabrus adspersus & & & & & -14.29 & 1 & 10.82 & 1 & & & & & 1 \\
\hline \multicolumn{14}{|l|}{ Zooplankton } \\
\hline Acartia tonsa (adults) & $-20.31 \pm 0.90$ & 4 & $7.08 \pm 2.45$ & 4 & & & & & & & & & 1 \\
\hline Acartia tonsa (copepodites) & $-20.33 \pm 0.51$ & 2 & $5.37 \pm 0.39$ & 2 & -22.02 & 1 & 7.03 & 1 & $-21.59 \pm 0.16$ & 4 & $6.17 \pm 0.50$ & 4 & 1 \\
\hline Acartia tonsa (nauplii) & $-20.19 \pm 0.42$ & 4 & $6.06 \pm 1.41$ & 4 & $-21.59 \pm 0.22$ & 6 & $7.20 \pm 0.39$ & 6 & $-21.00 \pm 0.27$ & 5 & $7.44 \pm 0.06$ & 5 & 1 \\
\hline Mnemiopsis leidyi & $-20.19 \pm 0.50$ & 3 & $11.33 \pm 0.45$ & 3 & $-20.25 \pm 0.64$ & 3 & $10.27 \pm 2.14$ & 3 & -18.74 & 1 & 7.03 & 1 & 1 \\
\hline Polychaete larvae & & & & & $-18.90 \pm 0.30$ & 4 & $8.63 \pm 0.77$ & 4 & $-19.77 \pm 0.16$ & 3 & $7.82 \pm 0.98$ & 3 & 1 \\
\hline
\end{tabular}

values in the water column and in benthic species due to nutrient supply and to trophic position.

\section{Coupling of different producer and consumer taxa to land-derived $\mathbf{N}$ sources}

The $\delta^{13} \mathrm{C}$ and $\delta^{15} \mathrm{~N}$ signatures of producers and consumers varied considerably among taxonomic groups, individual species, and across estuaries (Table 1). Distribution of species differed among the estuaries, with some species not present in all 3 estuaries. Overall, $\delta^{13} \mathrm{C}$ values of different species varied within estuaries due to their $\mathrm{C}$ source, but the values did not change across estuaries within the same species. $\delta^{15} \mathrm{~N}$ values of different species varied within estuaries and across estuaries within the same species, increasing from low to high $\mathrm{N}$ load.

The $\delta^{15} \mathrm{~N}$ isotopic signatures of macrophytes, sediment, and POM significantly increased with $\mathrm{N}$ load (Fig. 2a,c,e) and with the percent contribution of wastewater to total $\mathrm{N}$ load (Fig. 2b,d,f). These relationships suggest that these specific components of the estuarine ecosystems appear coupled to land use on the watersheds emptying into the area in which they were sampled. In addition, the regression slopes for macrophytes, sediment, and POM all differed significantly, with macrophytes showing the largest slope response to increasing $\mathrm{N}$ loads, followed by sediment, and then POM (Table 2). This result indicates that macrophytes may be the most sensitive indicator of eutrophication that could be used in monitoring landderived $\mathrm{N}$ load.

All taxonomic groups of benthic invertebrates and fishes consistently and significantly increased in $\delta^{15} \mathrm{~N}$ isotopic signatures with increasing $\mathrm{N}$ load. This remarkably consistent response of all these taxa indicates that they were well coupled to the watersheds and estuaries in which they were collected (Fig. 3). The responses of polychaetes, bivalves, decapods, and teleosts were the most pronounced (Fig. 3, Table 3). Polychaetes showed the greatest variation in $\delta^{15} \mathrm{~N}$ values within each estuary, with a range from 4 to $10 \%$ in Sage Lot Pond and 6 to $12 \%$ in Childs River (Fig. 3a). This variation is probably derived from the variation in feeding types among polychaetes (Appendix 1).

The teleost $\delta^{15} \mathrm{~N}$ signatures were much heavier overall than those of all other groups (Fig. 3h). There was considerable variation in fish $\delta^{15} \mathrm{~N}$ signatures, probably associated to temporal and spatial variability for some species, flexible food habits (Davenport \& Bax 2002), and inclusion of different feeding types (e.g. the predator Menidia menidia and the grazer Cyprinodon variegatus) among the fishes.

In strong contrast, the isotopic signature of zooplanktonic organisms did not vary with $\mathrm{N}$ load (Fig. 4). The range in $\delta^{15} \mathrm{~N}$ of zooplankton was approximately 3 to $13 \%$. Adults, copepodids, or nauplii of Acartia tonsa, 

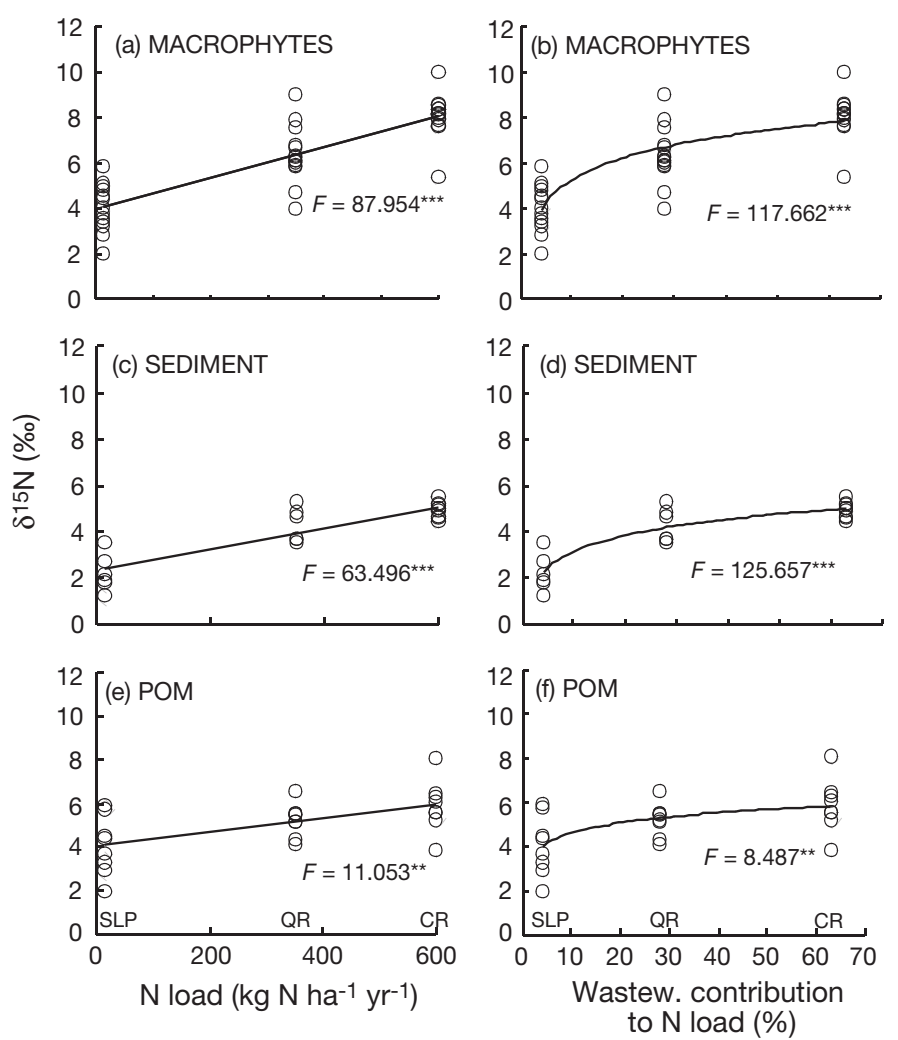

Fig. 2. $\delta^{15} \mathrm{~N}$ of macrophytes, sediment, and particulate organic matter (POM) vs. $(\mathrm{a}, \mathrm{c}, \mathrm{d}) \mathrm{N}$-loading rate and $(\mathrm{b}, \mathrm{d}, \mathrm{f})$ percentage of wastewater in Sage Lot Pond (SLP), Quashnet River (QR), and Childs Rivers (CR). Wastewater percentages are from McClelland et al. (1997). Regression analysis using $\mathrm{N}$ load generates: for macrophytes $y=x \times 0.007+3.956$, for sediment $y=x \times 0.005+2.276$, and for POM $y=x \times 0.003+4.053$; using wastewater percentage generates: for macrophytes $y=$ $2.911 \times \ln (x)-3.754$, for sediment $y=1.880 \times \ln (x)-2.646$, and for POM $y=1.322 \times \ln (x)+0.560$. Asterisks indicate significant regressions, $F$-test $\left({ }^{* * *} \mathrm{p}<0.001,{ }^{* *} \mathrm{p}<0.010\right)$

the most abundant copepod species, did not show any relationship between $\delta^{15} \mathrm{~N}$ values and $\mathrm{N}$ load. In addition, we did not find any pattern in signatures among the different copepod life stages. In Sage Lot Pond, adult $A$. tonsa were heavier, while nauplii and copepodids were lighter. In Quashnet River, adults had a lighter signature than copepodids or nauplii. Fortuitously, there were no adult copepods in our samples from Childs River, and polychaete larvae were not found in Sage Lot Pond. There was, however, no significant increment in $\delta^{15} \mathrm{~N}$ of polychaete larvae from samples collected in Quahsnet River and Childs River. Therefore, zooplankton in our estuaries appear to be largely uncoupled to the $\mathrm{N}$ entering from land-derived sources, in strong contrast to what we found so consistent for benthos.

The uncoupling of zooplankton from watershed influences in Waquoit Bay estuaries is not a straight-
Table 2. Results of $t$-tests comparing pairs of regression slopes constructed using $\delta^{15} \mathrm{~N}$ of primary producers vs. $\mathrm{N}$ loads from different estuaries of Waquoit Bay (data in Fig. 2)

\begin{tabular}{|lccc|}
\hline Comparison & df & $t$ & $p$ \\
\hline Macrophytes vs. POM & 60 & 18.5 & $<0.001$ \\
Macrophytes vs. Sediment & 57 & 22.0 & $<0.001$ \\
Sediment vs. POM & 41 & 5.0 & $<0.001$ \\
\hline
\end{tabular}

Table 3. Results of $t$-tests comparing linear regression slopes constructed using $\delta^{15} \mathrm{~N}$ data of taxa vs. $\mathrm{N}$ loads from different estuaries of Waquoit Bay (data in Fig. 3)

\begin{tabular}{|c|c|c|c|c|}
\hline Comparison & & $\mathrm{df}$ & $t$ & $\mathrm{p}$ \\
\hline \multirow[t]{7}{*}{ Polychaeta } & vs. Holothuroidea & 39 & 2.833 & $<0.001$ \\
\hline & vs. Gastropoda & 39 & 0.583 & 0.169 \\
\hline & vs. Bivalvia & 57 & 0.833 & 0.101 \\
\hline & vs. Decapoda & 58 & 0.750 & 0.004 \\
\hline & vs. Amphipoda & 42 & 0.667 & 0.117 \\
\hline & vs. Isopoda & 39 & 2.833 & 0.081 \\
\hline & vs. Teleostei & 76 & 0.417 & 0.320 \\
\hline \multirow[t]{6}{*}{ Holothuroidea } & vs. Gastropoda & 14 & 2.050 & $<0.001$ \\
\hline & vs. Bivalvia & 32 & 2.200 & 0.004 \\
\hline & vs. Decapoda & 33 & 1.250 & 0.030 \\
\hline & vs. Amphipoda & 17 & 1.300 & 0.062 \\
\hline & vs. Isopoda & 14 & 0 & 1.000 \\
\hline & vs. Teleostei & 51 & 1.450 & 0.030 \\
\hline \multirow[t]{5}{*}{ Gastropoda } & vs. Bivalvia & 32 & 0.176 & 0.787 \\
\hline & vs. Decapoda & 33 & 0.941 & 0.054 \\
\hline & vs. Amphipoda & 17 & 0.882 & 0.152 \\
\hline & vs. Isopoda & 14 & 2.412 & 0.011 \\
\hline & vs. Teleostei & 51 & 0.706 & 0.236 \\
\hline \multirow[t]{4}{*}{ Bivalvia } & vs. Decapoda & 51 & 3.167 & $<0.001$ \\
\hline & vs. Amphipoda & 35 & 3.000 & $<0.001$ \\
\hline & vs. Isopoda & 32 & 7.333 & 0.088 \\
\hline & vs. Teleostei & 69 & 2.500 & $<0.001$ \\
\hline \multirow[t]{3}{*}{ Decapoda } & vs. Amphipoda & 36 & 0.111 & 0.620 \\
\hline & vs. Isopoda & 33 & 2.778 & 0.264 \\
\hline & vs. Teleostei & 69 & 0.444 & 0.049 \\
\hline \multirow[t]{2}{*}{ Amphipoda } & vs. Isopoda & 16 & 3.714 & 0.295 \\
\hline & vs. Teleostei & 53 & 0.429 & 0.677 \\
\hline Isopoda & vs. Teleostei & 51 & 2.841 & 0.232 \\
\hline
\end{tabular}

forward feature. We know, for example, that female Acartia tonsa respond to the relative availability of food in the different Waquoit Bay estuaries, and increase their egg production in proportion to $\mathrm{N}$ load (Cubbage et al. 1999). Nonetheless, this response did not translate into a parallel effect on copepod abundances (Lawrence et al. 2004), nor, which is more relevant to the present paper, on isotopic signatures (Fig. 4). The uncoupling from the influence of watersheds shown by A. tonsa may be related to the short residence time of water in Waquoit Bay (Valiela et al. 2001, Lawrence et al. 2004). The estimated residence time in these estuaries is $\sim 2 \mathrm{~d}$ (Valiela et al. 2004), 


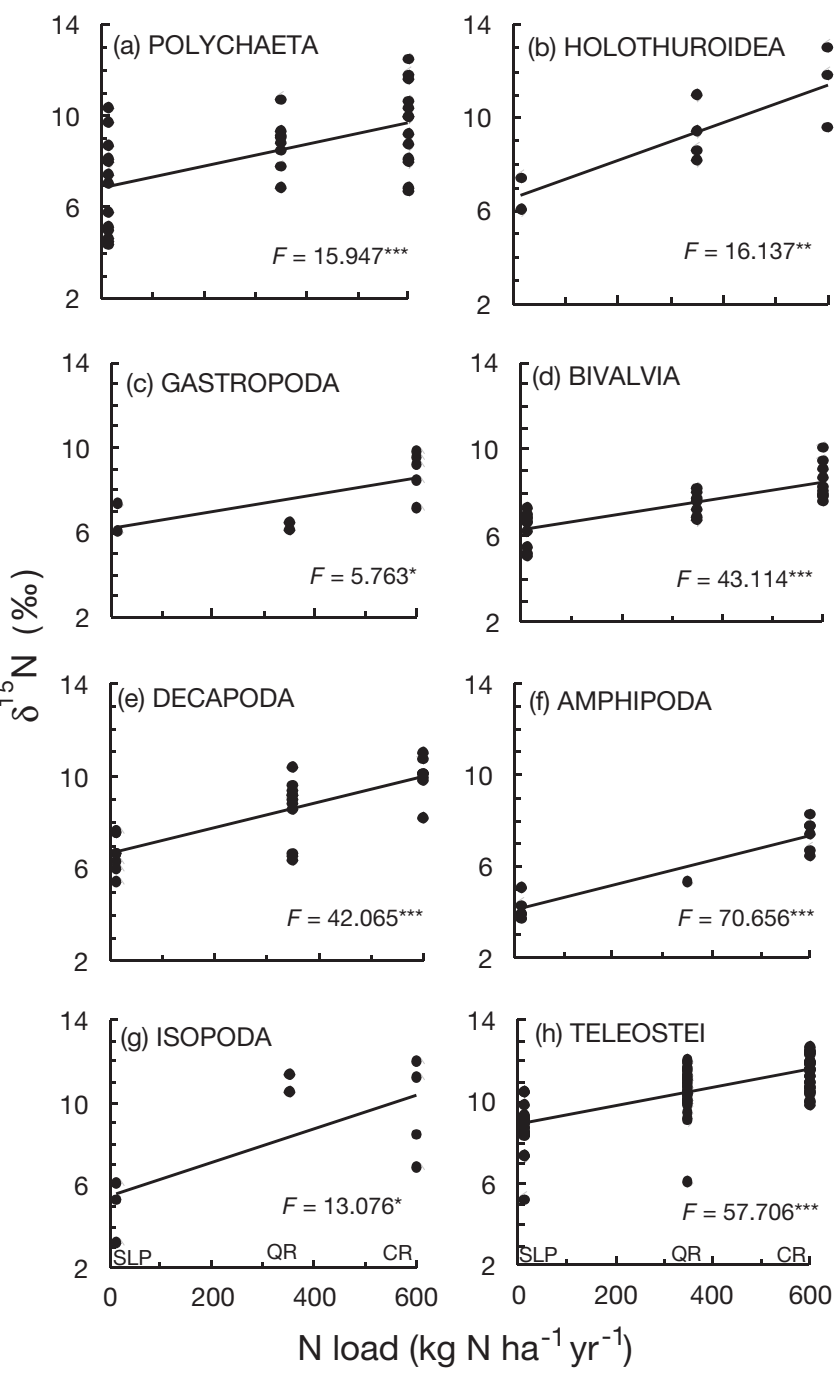

Fig. 3. $\delta^{15} \mathrm{~N}$ of organisms grouped by major taxa vs. N-loading rate in Sage Lot Pond (SLP), Quashnet River (QR), and Childs Rivers (CR). Asterisks indicate significant linear regressions, $F$-test $\left({ }^{* * *} \mathrm{p}<0.001,{ }^{* *} \mathrm{p}<0.010,{ }^{*} \mathrm{p}<0.050\right)$

which may be too brief for plankton to take advantage of the available food supply and increase their abundances. The short residence time may not be long enough either to allow zooplankton to assimilate the specific isotopic signature associated with specific watersheds. The uncoupling of copepods from landderived $\mathrm{N}$ loading occurs in spite of clear evidence that phytoplankton do acquire nitrogen signatures characteristic of each estuary (J. K. York, I. Valiela \& D. J. Repeta unpubl. data). Plankton feeding in one estuary may find themselves in altogether different places $2 \mathrm{~d}$ later, moved by tide and other hydrodynamic advection. This means that the plankton found at any one time may be a mixture of specimens that have experienced quite different feeding and geographic histories and, consequently, bear quite variable isotopic ratios.
Our data consistently demonstrate that primary producers, benthic invertebrates, and fishes were clearly coupled to the watersheds and estuaries in which they were found. In contrast, zooplanktonic organisms may be more subject to advective movements so that they were not as coupled.

\section{Coupling of trophic groups to land-derived $\mathrm{N}$ sources}

All trophic groups examined consistently increased their $\delta^{15} \mathrm{~N}$ signatures with $\mathrm{N}$ load (Fig. 5). Grazers showed an increment in $\delta^{15} \mathrm{~N}$ from low to high $\mathrm{N}$ loaded estuaries similar to that showed by macrophytes ( $\mathrm{df}=10, t=2.634, \mathrm{p}=0.292$ ), but they did not increase their signature compared to the macrophytes (ANCOVA: $F=0.606, p=0.440$; Fig. $5 \mathrm{a}$ ) nor to Cladophora vagabunda (ANCOVA: $F=2.563, \mathrm{p}=$ 0.133 ). This is puzzling, but suggests that these grazers were not strictly feeding on macrophytes. The Waquoit Bay food web seems to lack strict herbivores. In fact, we know, for example, that Cyprinodon variegatus, probably the most herbivorous species of fish on our list, also feeds on detritus and meiofauna (Werme 1981). Amphipod diets are largely algae (Hauxwell et al. 1998), but also include detritus (Zimmerman et al. 1979), so that the isotopic signature of 'grazers' could be lower due to consumption of detritus and sediment.

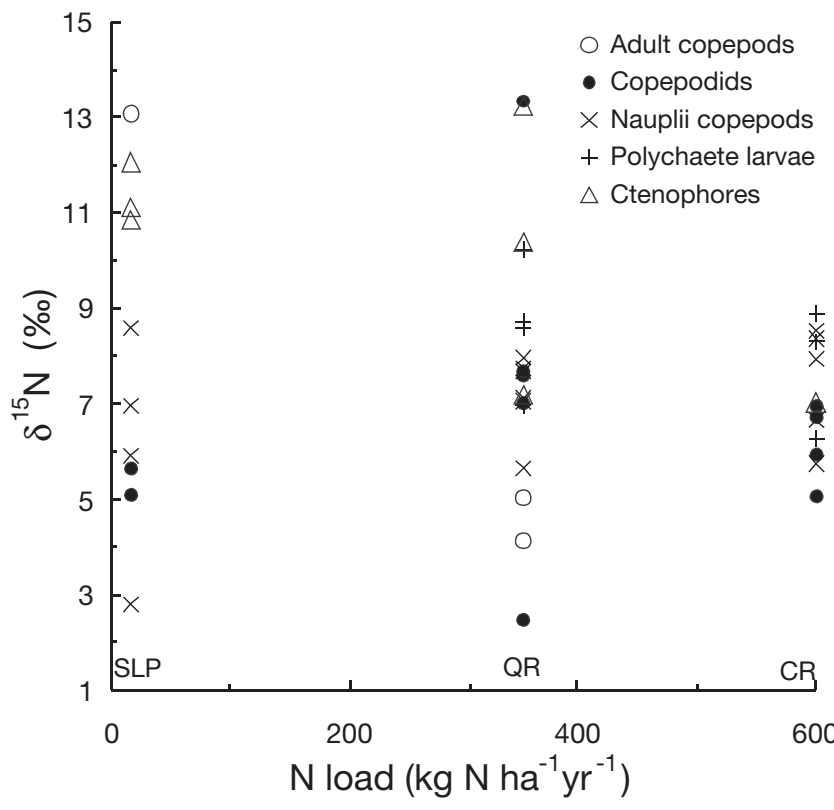

Fig. 4. $\delta^{15} \mathrm{~N}$ of zooplanktonic organisms vs. N-loading rate in Sage Lot Pond (SLP), Quashnet River (QR), and Childs Rivers (CR). Adults, copepodids and nauplii are Acartia tonsa. Regression analysis generates: for adults $\mathrm{p}=0.590, \mathrm{r}^{2}=0.219$; for copepodids $\mathrm{p}=0.103, \mathrm{r}^{2}=0.004$; for polychaete larvae $\mathrm{p}=$ $0.109, r^{2}=0.211$; and for ctenaphores $p=0.171, r^{2}=0.205$ 

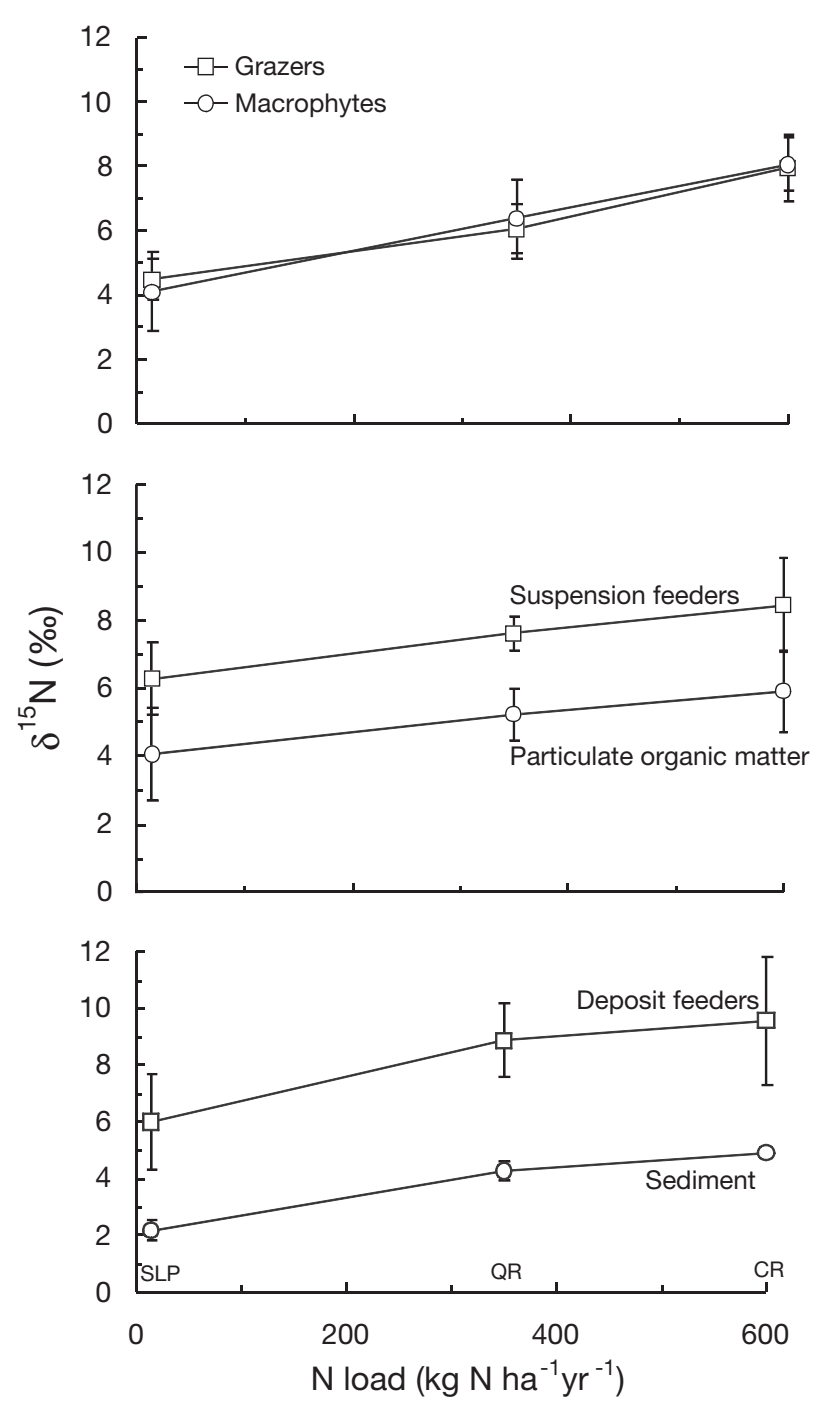

Fig. $5 . \delta^{15} \mathrm{~N}$ (means $\pm \mathrm{SE}$ ) of primary producers and consumers vs. N-loading rate in Sage Lot Pond (SLP), Quashnet River (QR), and Childs Rivers (CR). Here and and in other figures grazers are: Cyprinodon variegatus, Cymadusa compta, Microdeutopus gryllotalpa, Erichsonella filiformis, and Gammarus mucronatus; suspension feeders are: Crepidula fornicata, Polycirrus eximius, Argopecten irradians, Geukensia demissa, Mercenaria mercnaria, Mya arenaria, Molgula manhattensis, and Sabellaria vulgaris; deposit feeders are: Sclerodactyla briareus, Leptosynapta tenuis, Tharyx acutus, Cyrratulus grandis, Orbinia ornata, Heteromastus filiformis, Notomastus latericeus, Neanthes succinea, Pectinaria gouldii, and Clymenella torquata

The nearly similar isotopic signatures of macrophytes and the presumptive herbivores suggest that our 'herbivores' must use food items whose signatures are lighter than those of macrophytes to the extent that they mask the 2 to $4 \%$ fractionation to be expected of consumers of the macrophytes.

$\delta^{15} \mathrm{~N}$ signatures of suspension feeders, excluding the zooplankton taxa, and their POM food increased as $\mathrm{N}$ load increased (Fig. 5b). The slopes were similar ( $\mathrm{df}=18, t=2.445, \mathrm{p}=0.623$ ), but, as evident in the $y$ intercepts, the isotopic signature of the suspension feeders was enriched in ${ }^{15} \mathrm{~N}$ by $2.3 \%$, compared with the POM values (ANCOVA: $F=15.838$, $p<0.001$ ). Deposit feeders and sediment $\delta^{15} \mathrm{~N}$ signatures also increased as $\mathrm{N}$ load increased (Fig. 5c), with similar slopes ( $\mathrm{df}=25, t=3.361, \mathrm{p}=0.264$ ). The enrichment in ${ }^{15} \mathrm{~N}$ in deposit feeders was $3.9 \%$ relative to the signature in the sediment along the N-load gradient (ANCOVA: $F=30.241, \mathrm{p}<0.001$; Fig. 5c). These results suggest that both suspension and deposit feeders show signatures corresponding to the 2 to $4 \%$ o fractionation expected of trophic steps (Fig. 6). Thus, the trophic groups found in the benthos are clearly coupled to the watershed that input $\mathrm{N}$ into the estuary, and, to their food sources.

$\delta^{15} \mathrm{~N}$ signatures of benthic-associated predators increased as $\mathrm{N}$ load increased, in contrast to the $\delta^{15} \mathrm{~N}$ of zooplanktivore predators (Fig. 7). Benthic predators, such as polychaetes and crabs, were clearly coupled to the watersheds and estuaries, and reflected the isotopic signatures of their prey. Pelagic predators that actively fed on benthic prey, detritus, or plants, like the fishes, also assimilated the specific $\delta^{15} \mathrm{~N}$ signature of the respective estuary in which they were living. The time spent in the estuaries by these species seems to be long enough to assimilate the particular signature of each estuary, and they, being active swimmers, are not influenced by the residence time of the water, in contrast to planktonic organisms.

Some species we collected in Waquoit Bay have been described as zooplanktivores, but isotopic signa-

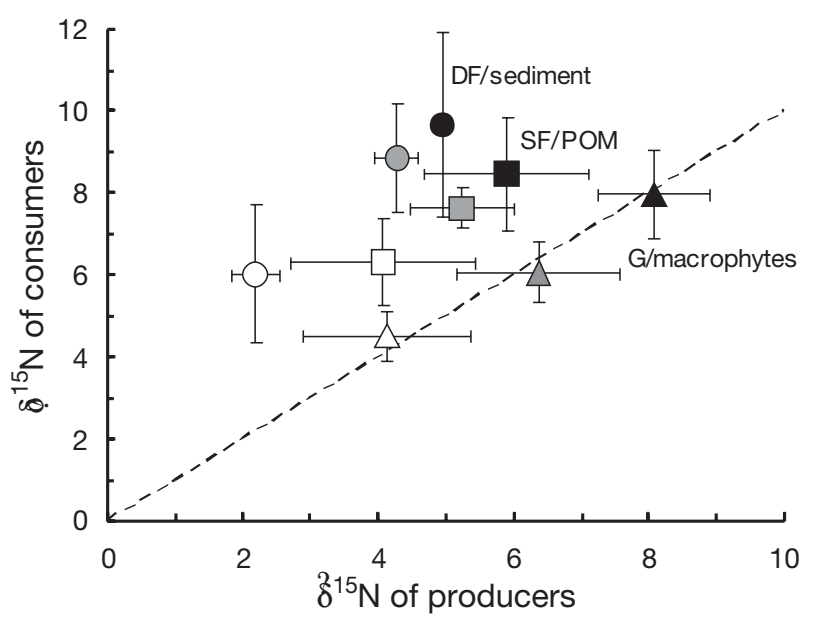

Fig. 6. $\delta^{15} \mathrm{~N}$ (means $\pm \mathrm{SE}$ ) of primary producers vs. those of consumers in Sage Lot Pond (open symbols), Quashnet River (grey symbols), and Childs River (black symbols). DF: deposit feeder (circles); SF: suspension feeders (squares); POM: particulate organic matter; G: grazers (triangles) 


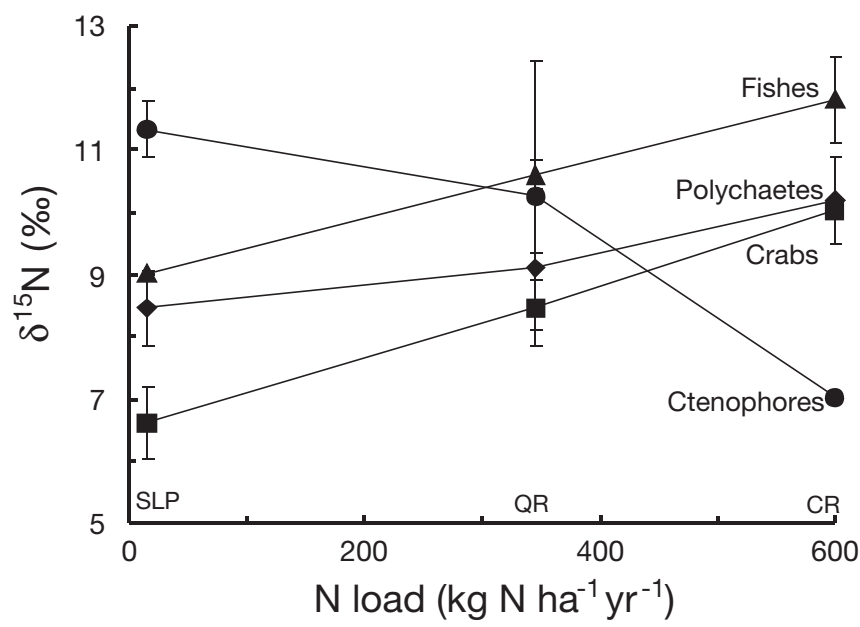

Fig. 7. $\delta^{15} \mathrm{~N}$ (means $\pm \mathrm{SE}$ ) of predators vs. N-loading rate in Sage Lot Pond (SLP), Quashnet River (QR), and Childs Rivers (CR). Fishes are: Menidia menidia, Fundulus majalis, Gobiosoma bosc, Tautogolabrus adspersus, and Anguilla rostrata; polychaetes are: Harmathoe imbricata, H. extenuata, Arabella iridicolor, and Podarke obscura; crabs are: Carcinus maenas, Rhitropanopeus harsii, and Callinectes sapidus

tures suggested otherwise. For example, Menidia menidia do feed on copepods, but they must also prey on benthic invertebrates (Griffin \& Valiela 2001). Benthic prey appeared to dominate the diet of $M$. menidia, since the $\delta^{15} \mathrm{~N}$ signatures of $M$. menidia follow the estuary signature (Table 1). M. menidia, although an active swimmer independent of water movements, nevertheless appears to remain within a single estuary long enough to assimilate the $\delta^{15} \mathrm{~N}$ signature of that estuary.

The only pelagic species that appears to be a strict zooplanktivore was the ctenophore Mnemiopsis leidyi. M. leidyi was uncoupled to the watersheds and the estuaries (Fig. 4), as well as to the zooplankton. This confirms that species that fed strictly within the water column food web were not affected by their watersheds.

So far we have referred to the $\delta^{15} \mathrm{~N}$ data, but the $\delta^{13} \mathrm{C}$ information also provides a perspective on the link between the benthic and pelagic components of the food webs. The carbon sources in our food web seem to be divided into 2 pathways (Fig. 8). Suspension feeders had lighter $\delta^{13} \mathrm{C}$ values than scavengers, grazers, predators, and deposit feeders, across all 3 estuaries (Fig. 8a). A reasonable explanation for this contrast may be that $\delta^{13} \mathrm{C}$ values are linked to the food source and by these feeding types. In these estuaries, macroalgae may be consumed by scavengers, grazers, predators, and deposit feeders (Fig. 8a). Evidence for this conclusion is given by the similarity in the $\delta^{13} \mathrm{C}$ signatures of these consumers and the macroalgal $\delta^{13} \mathrm{C}$ signature (Fig. 8b). In contrast, suspension feeders showed $\delta^{13} \mathrm{C}$ signatures that matched the $\delta^{13} \mathrm{C}$ values of POM (Fig. 8b). These results corroborate the $\delta^{15} \mathrm{~N}$ results and confirm that the benthic and water column parts of Waquoit Bay food webs are relatively independent.

The isotopic nitrogen data thus suggest that there are powerful links between land use on watersheds and those parts of the estuarine food webs that are associated with the benthos. Those components of the estuarine environment that are pelagic and feed on zooplankton seem to be uncoupled to the land and the benthos, as made evident by the $\mathrm{N}$ and $\mathrm{C}$ isotopic information.

The results of this study have applied and basic implications. In terms of application, it is apparent that taxa associated with the benthos are likely to be more appropriate indicators for monitoring eutrophication of estuarine waters. The basic implications of our results include the suggestion that understanding the structuring of estuarine food webs must involve partitioning the components into those whose controls might depend on external terrestrial factors and whose control may lie elsewhere.
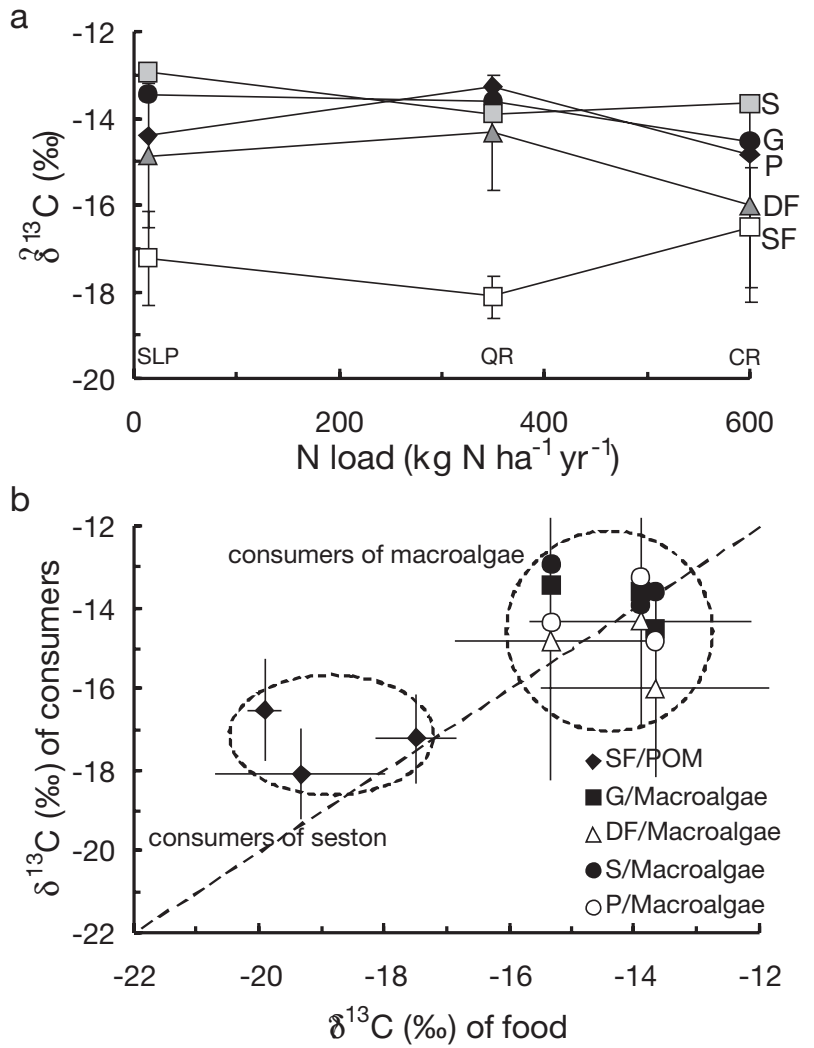

Fig. 8. $\delta^{13} \mathrm{C}$ of major trophic groups vs. (a) N-loading rate in Sage Lot Pond (SLP), Quashnet River (QR), and Childs Rivers (CR) and vs. (b) $\delta^{13} \mathrm{C}$ of presumed food sources. S: scavengers; G: grazers; P: predators; DF: deposit feeders; SF: suspension feeders; POM: particulate organic matter 
Acknowledgements. We thank David Lawrence for zooplankton identification, and Sophia Fox and Melissa Millman for field assistance. We are grateful for the comments and suggestions of Joanna York and 3 anonymous reviewers. P.M. was supported by a doctoral fellowship from CONICET (Consejo Nacional de Investigaciones Científicas y Técnicas, Argentina). This work was supported in part by ECOHAB Grant No. NA16OP2728. This is ECOHAB Publication No. 124.

\section{LITERATURE CITED}

Carmichael RH (2003) The effects of eutrophication on Mya arenaria and Mercenaria mercenaria: growth, survival, and physiological responses to changes in food supply and habitat across estuaries receiving different $\mathrm{N}$ loads. $\mathrm{PhD}$ dissertation, Boston University Marine Program, Woods Hole, MA

Chambers R (1981) Seasonal feeding and distribution of Palaemonetes pugio and $P$. vulgaris in Great Sippewissett salt marsh. Biol Bull (Woods Hole) 161:324

Chester CM (1993) Comparative feeding biology of Acteocinea candiculata (Say, 1826) and Haminoea solitaria (Say, 1822) (Ophistobranchia: Cephalaspidea). Am Malacol Bull 10:93-101

Cloern JE (2001) Our evolving conceptual model of the coastal eutrophication problem. Mar Ecol Prog Ser 210: 223-253

Costello JH, Loftus R, Waggett R (1999) Influence of prey detection on capture success for the ctenophore Mnemiopsis leidyi feeding upon adult Acartia tonsa and Oithona colcarva copepods. Mar Ecol Prog Ser 191: 207-216

Cruz Rivera E, Hay ME (2000) The effect of the diet mixing on consumer fitness: macroalgae, epiphytes, and animal matter as food for marine amphipods. Oecologia 123:252-264

Cubbage A, Lawrence D, Tomasky G, Valiela I (1999) Relationship of reproductive output in Acartia tonsa, chlorophyll concentration, and land-derived nitrogen loads in estuaries of Waquoit Bay, Massachusetts. Biol Bull (Woods Hole) 197:194-195

Dahlberg M, Conyers J (1979) An ecological study of Gobiosoma bosc and G. ginsburgi (Pisces, Gobiidae) on the Georgia coast. Fish Bull (Wash DC) 71:279-287

Daly JM (1973) The ability to locate a source of vibrations as a prey-capture mechanism in Harmothoe imbricata (Annelida Polychaeta). Mar Behav Physiol 14:305-322

Davenport SR, Bax NJ (2002) A trophic study of a marine ecosystem off southeastern Australia using stable isotopes of carbon and nitrogen. Can J Fish Aquat Sci 59:514-530

Denton AB, Chapman ARO (1991) Feeding preferences of gammarid amphipods among four species of Fucus. Mar Biol 109:503-506

Devore JL (2000) Probability and statistics for engineering and the sciences, 5th edn. Duxbury Press, Belmont, CA

Elner RW (1981) Diet of green crab Carcinus maenas (L.) from Port Hebert, southwestern Nova Scotia. J Shellfish Res 1: 89-94

GESAMP (Joint Group of Experts on the Scientific Aspects of Marine Pollution) (1990) The state of the marine environment. Rep and Stud 39. United Nations Environmental Programme, Nairobi

Gibbons MC (1984) Aspects of predation by the crabs Neopanopeus syai, Ovalipes ocellatus, and Pagurus longicarpus on juvenile hard clams Mercenaria mercenaria. PhD dissertation, State University of New York, Buffalo, NY

Grall J, Chauvaud L (2002) Marine eutrophication and ben- thos: the need for new approaches and concepts. Global Change Biol 8:813-830

Griffin M, Valiela I (2001) $\delta^{15} \mathrm{~N}$ isotope studies of life history and trophic position of Fundulus heteroclitus and Menidia menidia. Mar Ecol Prog Ser 214:299-305

Hauxwell J, McClelland J, Valiela I (1998) Relative importance of grazing and nutrient controls of macroalgal biomass in three temperate shallow estuaries. Estuaries 21: 347-360

Hsueh P, McClintock JB, Hopkins TS (1992) Comparative study of the diets of the blue crabs Callinectes similis and C. sapidus from a mud-bottom habitat in Mobile Bay, Alabama. J Crustac Biol 12:615-619

Huang SC, Kreeger DA, Newell RIE (2003) Seston available for the ribbed mussel (Geukensia demissa) in a North American, mid-Atlantic saltmarsh. Estuar Coast Shelf Sci 56:561-571

Hudon C (1983) Selection of unicellular algae by the littoral amphipods Gammarus oceanicus and Caliopius laevisculus (Crustacea). Mar Biol 78:59-67

Kleppel GS (1993) On the diet of calanoid copepods. Mar Ecol Prog Ser 99:183-195

Kozloff EN (1990) Invertebrates. University of Washington Sounder College Publishing, New York

Lawrence D, Valiela I, Tomasky G (2004) Estuarine calanoid copepod abundance in relation to season, salinity, and land-derived nitrogen loading, Waquoit Bay, MA. Estuar Coast Shelf Sci 61:547-557

Lesser MP, Shumway SE, Cucci T, Smith J (1992) Impact of fouling organisms on mussel rope culture: interspecific competition for food among suspension-feeding invertebrates. J Exp Mar Biol Ecol 165:91-102

Lu R, Ji R, Zon Y, Xia B, Li R, Zhu M (2000) A study of the feeding of three bivalves. Acta Oceanol Sin 22:306-312

Luckenbach MW, Orth RJ (1999) Effects of a deposit-feeding invertebrate on the entrapment of Zostera marina L. seeds. Aquat Bot 62:235-247

Martin D, Pinedo S, Sarda R (2000) Distribution patterns and trophic structure of soft-bottom polychaete assemblages in a north-western Mediterranean shallow-water bay. Ophelia 53:1-17

McAloon KM, Mason RP (2003) Investigations into the bioavailability and bioaccumulation of mercury and other trace metals to the sea cucumber, Sclerodactyla briareus, using in vitro solubilization. Mar Pollut Bull 46:1600-1608

McClelland JM, Valiela I (1998a) Changes in food web structure under the influence of increased anthropogenic nitrogen inputs to estuaries. Mar Ecol Prog Ser 168:259-271

McClelland JW, Valiela I (1998b) Linking nitrogen in estuarine producers to land-derived sources. Limnol Oceanogr 43:577-585

McClelland JW, Valiela I, Michener RH (1997) Nitrogenstable isotope signatures in food webs: a record of increasing urbanization in coastal watersheds. Limnol Oceanogr 42:930-937

Michener RH, Schell DM (1994) Stable isotope ratios as tracers in marine aquatic food webs. In: Lajtha $K$, Michener RA (eds) Stable isotopes in ecology and environmental science. Blackwell Scientific Publications, Oxford, p 138-157

Milke ML, Kennedy VS (2001) Mud crab (Xanthidae) in Chesapeake Bay: claw characteristics and predation on epifaunal bivalves. Invertebr Biol 120:67-77

National Research Council (1994) Priorities for coastal science. National Academy Press, Washington, DC

Nero L (1976) The natural history of the naked goby Gobiosoma bosc (Perciformes: Gobiidae). MS thesis, Old Dominion University, Norfolk, VA 
Neter J, Wasserman W, Kutner MH (1985) Applied linear statistical models, 2nd edn. RD Irwin, Homewood, IL

Nixon SW (1995) Coastal marine eutrophication: a definition, social causes and future concerns. Ophelia 41:199-219

Noyes GS (1980) The biology of Aglaophamus neotenus (Polychaeta: Nephtyidae), a new species from Maine and Canada. Biol Bull (Woods Hole) 158:103-117

Oakes MJ, Haven DS (1971) Some predators of polyps of Chrysaora quinqueciarrha (Scyphozoa, Semaeostomeae) in the Chesapeake Bay. Va J Sci 22:45-46

Ojeda FP, Dearborn JH (1991) Feeding ecology of benthic mobile predators: experimental analyses of their influence in rocky subtidal communities of the Gulf of Maine. J Exp Mar Biol Ecol 149:13-44

Pardo EV, Dauer DM (2003) Particle size selection in individuals from epifaunal versus infaunal populations of the nereidid polychaete Neanthes succinea (Polychaeta: Nereididae). Hydrobiologia 496:355-360

Petersen R, Svane I (2002) Filtration rate in seven Scandinavian ascidians: implication of the morphology of the gill sac. Mar Biol 140:397-402

Peterson BJ, Fry B (1987) Stable isotopes in ecosystems studies. Annu Rev Ecol Syst 18:293-320

Plante CJ, Shriver AG (1998) Patterns of differential digestion of bacteria in deposit feeders: a test of resource partitioning. Mar Ecol Prog Ser 163:253-258

Ray GL (1982) Ecology of benthic macroinvertebrates in two New Jersey salt marsh waterways. PhD dissertation, Rutgers University, New Brunswick, NJ

Rouse GW, Pleijel F (2001) Polychaetes. Oxford University Press, New York

Sanchez-Gonzales S, Ruiz-Campos G, Contreras-Balderas S (2001) Feeding ecology and habitat of the three spine stickleback, Gasterosteus aculeatus microcephalus, in a remnant population of northwestern Baja California, Mexico. Ecol Freshwat Fish 10:191-197

Shriver AC, Carmichael RH, Valiela I (2002) Growth, condition, reproductive potential, and mortality of bay scallops, Argopecten irradians, in response to eutrophic-driven changes in food resources. J Exp Mar Biol Ecol 279:21-40

Sokal RR, Rohlf FJ (2003) Biometry: the principles and practice of statistics in biological research, 3rd edn. WH Freeman \& Company, New York

Stachowicz JJ, Hay ME (1999) Reducing predation through chemically mediated camouflage: indirect effects of plant defenses on herbivores. Ecology 80:495-509

Stehlik LL, Meise CJ (2000) Diet of winter flounder in a New Jersey estuary: ontogenetic change and spatial variation. Estuaries 23:381-391
Tettelbach ST (1985) Temporal variability of crab predation on northern bay scallops, Argopecten irradians irradians. Estuaries 8:79

Valderhang VA (1985) Population structure and production of Lumbrinereis fragilis (Polychaeta: Lumbrinereide) in the Oslo fjord (Norway) with a note on metal content on jaws. Mar Biol 86:203-211

Valiela I, McClelland J, Hauxwell J, Behr PJ, Hersh D, Foreman K (1997) Macroalgal blooms in shallow estuaries: control and ecophysiological and ecosystem consequences. Limnol Oceanogr 42:1105-1118

Valiela I, Geist M, McClelland J, Tomasky G (2000) Nitrogen loading from watersheds to estuaries: verification of the Waquoit Bay Nitrogen Loading Model. Biogeochemistry 49:277-293

Valiela I, Bowen JL, Cole ML, Kroeger KD, Lawrence D, Pabich WJ, Tomasky G, Mazzilli S (2001) Following up on a Margalevian concept: interaction and exchanges among adjacent parcels of coastal landscapes. Sci Mar 65: 215-229

Valiela I, Mazzilli S, Bowen JL, Kroeger KD, Cole ML, Tomasky G, Isaji T (2004) ELM, an estuarine nitrogen loading model: formulation and verification of predicted concentrations of dissolved inorganic nitrogen. Water Air Soil Pollut 157:365-391

Weiss ET, Carmichael RH, Valiela I (2002) The effect of nitrogen loading on the growth rates of quahogs (Mercenaria mercenaria) and soft-shell clams (Mya arenaria) through changes in food supply. Aquaculture 211:275-289

Werme C (1981) Resource partitioning in a salt marsh fish community. PhD dissertation, Boston University Marine Program, Woods Hole, MA

Whitlatch RB, Weinberg JR (1982) Factors influencing particle selection and feeding rate in the polychaete Cistenides (Pectinaria) gouldii. Mar Biol 71:33-40

Wigand C, Comeleo R, McKinney R, Thursby G, Chintala M, Charpentier M (2001) Outline of a new approach to evaluate ecological integrity of salt marshes. Hum Ecol Risk Assess 7:1541-1554

Wilcox JR, Jeffries HP (1974) Feeding habits of the sand shrimp Crangon septemspinosa. Biol Bull (Woods Hole) 146:424-434

Yelenik S, McClelland J, Valiela I (1996) Changes in N and C isotopes signatures of particulate organic matter and ribbed mussels in estuaries subject to different nutrient loading. Biol Bull (Woods Hole) 191:329-330

Zimmerman R, Gibson R, Harrington J (1979) Herbivory and detritivory among gammaridean amphipods from a Florida seagrass community. Mar Biol 54:41-47

Appendix 1. Trophic groups of the analyzed species based on literature data

\begin{tabular}{|lll|}
\hline Species & Trophic group & Source \\
\hline Amphipoda & & \\
Cymadusa compta & Grazer & Hauxwell et al. (1998) \\
Gammarus mucronatus & Grazer & Cruz Rivera \& Hay (2000), Zimmerman et al. (1979) \\
Gammarus oceanicus & Grazer/detrivore & Hudon (1983), Denton \& Chapman (1991) \\
Microdeutopus gryllotalpa & Grazer & Hauxwell et al. (1998) \\
Ascidiacea & & Peterson \& Svane (2002) \\
Molgula manhattensis & Suspension feeder & \\
Bivalvia & & Lu et al. (2000) \\
Argopecten irradians & Suspension feeder & Huang et al. (2003) \\
Geukensia demissa & Suspension feeder & \\
\hline
\end{tabular}


Appendix 1 (continued)

\begin{tabular}{|c|c|c|}
\hline Species & Trophic group & Source \\
\hline \multicolumn{3}{|l|}{ Bivalvia (continued) } \\
\hline Mercenaria mercenaria & Suspension feeder & Weiss et al. (2002) \\
\hline Mya arenaria & Suspension feeder & Weiss et al. (2002) \\
\hline \multicolumn{3}{|l|}{ Decapoda } \\
\hline Callinctes sapidus & Predator & Hsueh et al. (1992) \\
\hline Carcinus maenas & Predator & Elner (1981) \\
\hline Crangon septemspinosa & Predator/detritivore & Wilcox \& Jeffries (1974) \\
\hline Libinia dubia & Predator/grazer & Oakes \& Haven (1971), Stachowicz \& Hay (1999) \\
\hline Neopanopeus sayi & Predator/detritivore & Gibbons (1984) \\
\hline Pagurus longicarpus & Predator/detritivore & Gibbons (1984), Tettelbach (1985) \\
\hline Palaemonetes pugio & Predator/detritivore & Chambers (1981) \\
\hline Palaemonetes vulgaris & Predator/detritivore & Chambers (1981) \\
\hline Rhitropanopeus harssi & Predator & Milke \& Kennedy (2001) \\
\hline \multicolumn{3}{|l|}{ Gastropoda } \\
\hline Crepidula fornicata & Suspension feeder & Lesser et al. (1992) \\
\hline Haminoea solitaria & Detrivore & Chester (1993) \\
\hline Nassarius obsoletus & Oportunistic deposit feeder & Ray (1982) \\
\hline \multicolumn{3}{|l|}{ Holothuroidea } \\
\hline Leptosynapta tenuis & Deposit feeder & Plante \& Shriver (1998) \\
\hline Sclerodactyla briareus & Deposit feeder & McAloon \& Mason (2003) \\
\hline \multicolumn{3}{|l|}{ Isopoda } \\
\hline Cyathura polita & Detrivore & Ray (1982) \\
\hline Erichsonela filiformis & Grazer & Hauxwell et al. (1998) \\
\hline \multicolumn{3}{|l|}{ Polychaeta } \\
\hline Aglaophamus circinata & Predator/omnivore & Kozloff (1990), Noyes (1980) \\
\hline Arabella iridicolor & Predator & Rouse \& Pleijel (2001) \\
\hline Cirratulus grandis & Deposit feeder & Rouse \& Pleijel (2001) \\
\hline Clymenella torquata & Deposit feeder & Luckenbach \& Orth (1999) \\
\hline Eteone lactea & Predator/scavenger & Rouse \& Pleijel (2001) \\
\hline Glycera americana & Opportunistic deposit feeder & Ray (1982) \\
\hline Harmothoe extenuata & Predator & Rouse \& Pleijel (2001) \\
\hline Harmothoe imbricata & Predator & Daly (1973) \\
\hline Heteromastus filiformis & Deposit feeder & Ray (1982) \\
\hline Lumbrinereis fragilis & Predator & Valderhang (1985) \\
\hline Neanthes succinea & Deposit feeder & Pardo \& Dauer (2003) \\
\hline Notomastus latericeus & Deposit feeder & Martin et al. (2000) \\
\hline Orbinia ornata & Deposit feeder & Rouse \& Pleijel (2001) \\
\hline Pectinaria gouldii & Deposit feeder & Whitlatch \& Weinberg (1982) \\
\hline Podarke obscura & Predator & Kozloff (1990) \\
\hline Polycirrus eximius & Suspension feeder & Rouse \& Pleijel (2001) \\
\hline Sabellaraia vulgaris & Suspension feeder & Rouse \& Pleijel (2001) \\
\hline Tharyx acutus & Deposit feeder & Rouse \& Pleijel (2001) \\
\hline \multicolumn{3}{|l|}{ Teleostei } \\
\hline Anguilla rostrata & Predator & Werme (1981) \\
\hline Cyprinodon variegatus & Grazer & Werme (1981) \\
\hline Fundulus heteroclitus & Predator/detrivore & Werme (1981) \\
\hline Fundulus majalis & Predator & Werme (1981) \\
\hline Gasterosteus aculeatus & Predator & Sanchez-Gonzales et al. (2001) \\
\hline Gobiosoma bosc & Predator & Nero (1976), Dahlberg \& Conyers (1979) \\
\hline Menidia menidia & Predator & Griffin \& Valiela (2001) \\
\hline Pseudopleuronectes americanus & Predator & Stehlik \& Meise (2000) \\
\hline Tautogolabrus adspersus & Benthivore & Ojeda \& Dearborn (1991) \\
\hline \multicolumn{3}{|l|}{ Ctenophora } \\
\hline Mnemiosis leidyi & Zooplanktivore & Costello et al. (1999) \\
\hline \multicolumn{3}{|l|}{ Copepoda } \\
\hline Acartia tosa & Phyto-microzooplanktivore & Kleppel (1993) \\
\hline
\end{tabular}

